\title{
Cumulative Impact of HIV and Multiple Concurrent Human Papillomavirus Infections on the Risk of Cervical Dysplasia
}

\author{
David H. Adler, ${ }^{1}$ Melissa Wallace, ${ }^{2}$ Thola Bennie, ${ }^{2}$ Beau Abar, ${ }^{1}$ Tracy L. Meiring, \\ Anna-Lise Williamson, ${ }^{3,4}$ and Linda-Gail Bekker ${ }^{2}$ \\ ${ }^{1}$ Department of Emergency Medicine, University of Rochester, Rochester, NY 14642, USA \\ ${ }^{2}$ Desmond Tutu HIV Centre, Institute of Infectious Diseases \& Molecular Medicine, Faculty of Health Sciences, \\ University of Cape Town, Anzio Road, Observatory, Cape Town, South Africa \\ ${ }^{3}$ Institute of Infectious Diseases \& Molecular Medicine and Division of Medical Virology, Faculty of Health Sciences, \\ University of Cape Town, Anzio Road, Observatory, Cape Town, South Africa \\ ${ }^{4}$ National Health Laboratory Service, Groote Schuur Hospital, Cape Town, South Africa \\ Correspondence should be addressed to David H. Adler; david_adler@urmc.rochester.edu
}

Received 1 December 2015; Accepted 26 January 2016

Academic Editor: Finn S. Pedersen

Copyright (C) 2016 David H. Adler et al. This is an open access article distributed under the Creative Commons Attribution License, which permits unrestricted use, distribution, and reproduction in any medium, provided the original work is properly cited.

Infection with HIV is known to increase the risk of cervical cancer. In addition, evidence suggests that concurrent infection with multiple human papillomavirus (HPV) genotypes increases the risk of cervical dysplasia more than infection with a single HPV genotype. However, the impact of the combination of HIV coinfection and presence of multiple concurrent HPV infections on the risk of cervical dysplasia is uncertain. We compared the results of HPV testing and Pap smears between HIV-infected and HIVuninfected young women to assess the cumulative impact of these two conditions. We found that both HIV and the presence of multiple concurrent HPV infections are associated with increased risk of associated Pap smear abnormality and that the impact of these two risk factors may be additive.

\section{Introduction}

Coinfection with HIV has a significant impact on the natural history of high-risk human papillomavirus (HR-HPV) infections, the causative agents of cervical cancer. Women with HIV are more likely to be infected with HPV, and more likely to harbor multiple concurrent HPV infections (i.e., simultaneous infection with two or more HPV genotypes) [1]. While many HPV infections are transient, HIV-infected women are more likely to have persistent HPV infections $[2,3]$ which are associated with a greater incidence and progression of the precancerous lesions caused by HPV $[4,5]$. Worsening immunodeficiency correlates with an increased risk of advanced cervical dysplasia [6]. Invasive cervical cancer, an AIDS defining illness, is 2 to 22 times more likely to develop in HIV-infected women [7].

Multiple concurrent HPV infections have been associated with an increased risk of persistent HPV infection [8] and of developing precancerous cervical lesions $[9,10]$ compared to infection with a single HPV genotype. Simultaneous infection with HIV and multiple concurrent HPVs may increase the risk of developing precancerous lesions more than either condition on its own.

Cervical cancer is the number one cancer cause of years of life lost in the developing world [11] where over eighty-five percent of global cases and deaths from cervical cancer occur, with the very highest rates in Sub-Saharan Africa $[12,13]$. We compared the prevalence of multiple concurrent high-risk HPV infections between HIV-infected and HIV-uninfected South African young women and the association of these infections with cervical cytological abnormalities.

\section{Materials and Methods}

Between October 2013 and March 2015, we conducted a longitudinal study of $50 \mathrm{HIV}$-infected and $50 \mathrm{HIV}$-uninfected 
TABLE 1: Participant demographics and behavioral variables.

\begin{tabular}{|c|c|c|c|c|c|c|c|c|c|}
\hline & \multicolumn{4}{|c|}{ HIV-infected } & \multicolumn{4}{|c|}{ HIV-uninfected } & \multirow[b]{2}{*}{$p$} \\
\hline & $\begin{array}{c}\mathrm{M} \\
(\mathrm{SD})\end{array}$ & IQR & $f$ & $\%$ & $\begin{array}{c}\mathrm{M} \\
(\mathrm{SD})\end{array}$ & IQR & $f$ & $\%$ & \\
\hline Age at first visit & $\begin{array}{l}19.6 \\
(1.4)\end{array}$ & $19.0-21.0$ & & & $\begin{array}{l}18.4 \\
(1.4)\end{array}$ & $17.0-19.3$ & & & $<0.001$ \\
\hline Smoker at any time & & & & & & & & & 0.28 \\
\hline No & & & 43 & $88 \%$ & & & 47 & $94 \%$ & \\
\hline Yes & & & 6 & $12 \%$ & & & 3 & $6 \%$ & \\
\hline Lifetime sexual partners & & & & & & & & & 0.05 \\
\hline 1 & & & 11 & $22 \%$ & & & 4 & $8 \%$ & \\
\hline $2-5$ & & & 34 & $68 \%$ & & & 44 & $88 \%$ & \\
\hline$>5$ & & & 5 & $10 \%$ & & & 2 & $4 \%$ & \\
\hline Sexual partners in the last 6 months & & & & & & & & & 0.65 \\
\hline 1 & & & 48 & $96 \%$ & & & 47 & $94 \%$ & \\
\hline $2-5$ & & & 2 & $4 \%$ & & & 3 & $6 \%$ & \\
\hline Number of past pregnancies & & & & & & & & & 0.84 \\
\hline 0 & & & 33 & $66 \%$ & & & 34 & $68 \%$ & \\
\hline 1 & & & 15 & $30 \%$ & & & 15 & $30 \%$ & \\
\hline 2 & & & 2 & $4 \%$ & & & 1 & $2 \%$ & \\
\hline
\end{tabular}

young, sexually active, South African women. Cohort enrollment occurred sequentially until the goal of 50 participants in each group was met. Study participants were recruited through the Youth Centre in Masiphumelele and the Hannan Crusaid Clinic in Gugulethu, townships in the Cape Town area of South Africa. The Youth Centre in Masiphumelele serves the general adolescent population of the township, while the Hannan Crusaid Clinic specifically serves the HIV-infected population of Gugulethu. Demographic and behavioral variables of our cohort are presented in Table 1. Informed consent (age 18 years or older) or parental consent along with signed adolescent assent (age 16-17 years) was obtained from all participants. The Institutional Review Boards of the University of Rochester and the University of Cape Town approved this study.

Serial HPV DNA testing, utilizing self-collected specimens, was conducted approximately every six months for all participants. In addition, all study participants underwent baseline Pap smear testing. Most study participants underwent additional Pap testing during the follow-up period. Subsequent Pap testing (not included in this analysis) was conducted at varying intervals depending on HIV status and previous Pap result as per the guidelines of the Western Cape Province. Only the enrollment HPV and Pap test results are used in this analysis. HIV status was confirmed upon enrollment and HIV-uninfected participants underwent HIV testing at every study visit. Women with a history of HPV vaccination or cervical surgery were excluded.

Roche Diagnostics Linear Array HPV Test was used for all HPV genotyping. This kit identifies $37 \mathrm{HPV}$ genotypes including all oncogenic "high-risk" HPV genotypes (HR$\mathrm{HPV}$ ) as designated by the International Agency for Research on Cancer [14]. These 13 HR-HPV genotypes include types 16 , $18,31,33,35,39,45,51,52,56,58,59$, and 68. Specimens for HPV testing were obtained via self-sampling, performed in private, in which study participants were instructed to twirl a Dacron swab high in the vagina for 10 seconds. Specimens were then transported to the laboratory in Digene transport medium. The MagNA Pure Compact Nucleic Acid Isolation Kit (Roche) was used to extract DNA. All Pap smears were reported per the Bethesda system. Per laboratory protocol a pathologist reviewed all positive Pap smears. We defined any Pap not categorized as "negative," including atypical squamous cell of undetermined significance (ASCUS) to be abnormal.

Pearson $\chi^{2}$ tests for independence were used to compare groups, and Pearson and Spearman correlations were used to examine bivariate associations. All analyses were performed using IBM SPSS 22.0.

\section{Results}

Preliminary descriptive cross-sectional data from the initial 85 participants in this cohort have been previously published [15]. Among our final and complete cohort, we found an overall HPV prevalence rate of $64 \%$ and $\mathrm{HR}-\mathrm{HPV}$ prevalence rate of $38 \%$. Multiple concurrent HPV infections were found among $41 \%$ of participants and multiple concurrent HRHPV infections were found among $24 \%$. Since a wealth of research has shown cytological abnormalities to be driven by the presence of an HR-HPV infection, we examined Pap test results only among women with at least one such infection. The results indicated that having multiple concurrent HPV infections was associated with twice the likelihood of an associated Pap smear abnormality compared to having a single HPV infection (34\% versus 17\%), although this difference did not reach statistical significance $(p=0.15)$.

Overall, HIV-infected women had a much greater burden of HPV infection. HIV-infected participants had greater proportions of multiple concurrent HPV infections (60\% versus 
TABLE 2: HPV infections, Pap test results, and HIV status.

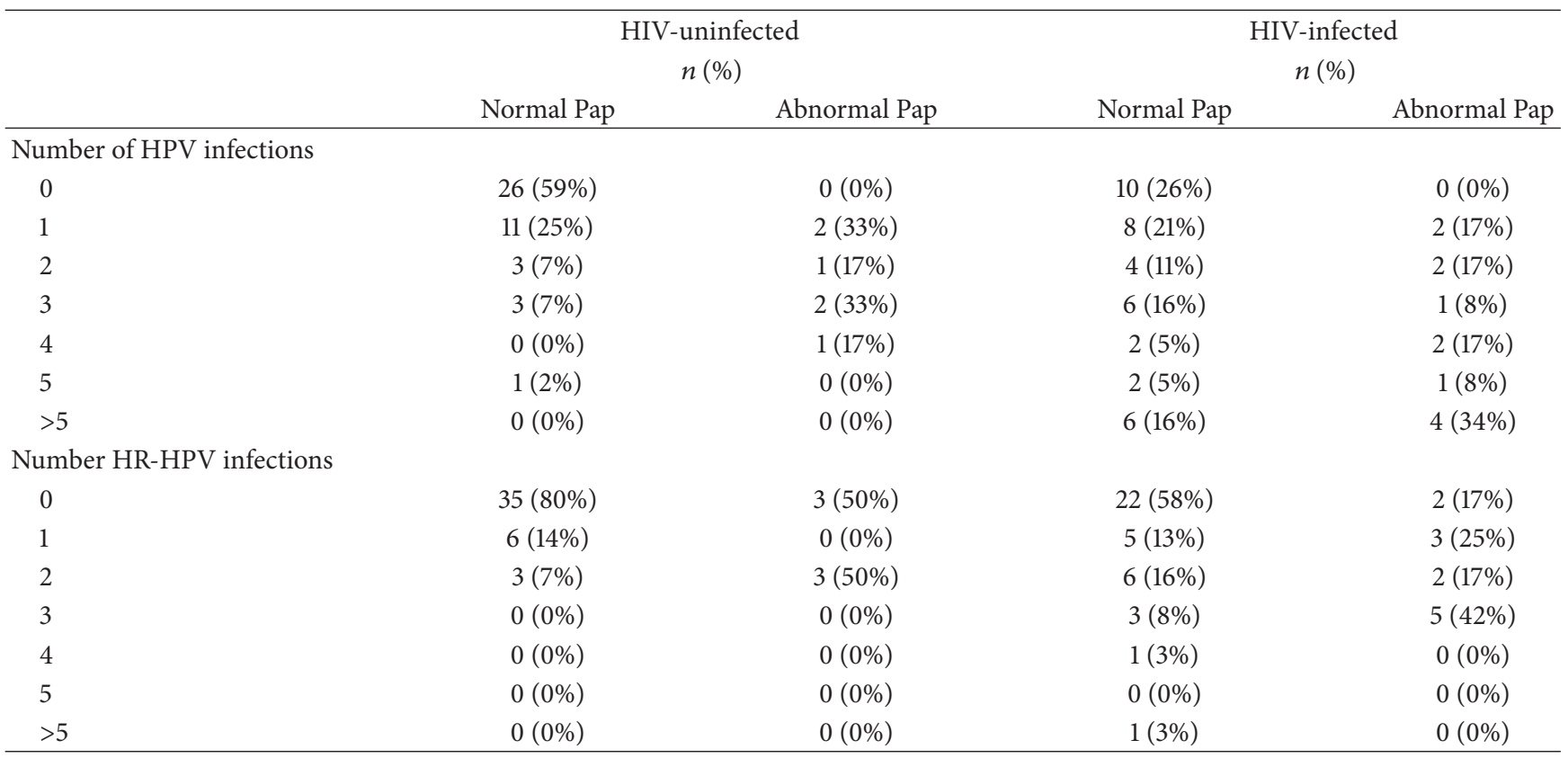

Note: number of HPV infections and number of HR-HPV infections were associated with age (Pearson $r=0.23, p=0.019 ; r=0.20, p=0.048$, resp.).

$22 \%, p<0.001)$ and multiple concurrent HR-HPV infections (36\% versus $12 \%, p=0.004$ ). The average number of HPV infections was 2.92 for the HIV-infected group compared to 0.90 for the HIV-uninfected group $(p<0.001)$. Likewise, the average number of HR-HPV infections was greater among those with HIV (1.16 versus $0.36, p=0.001)$. HIV-infected participants had a greater proportion of abnormal Pap smears at baseline (24\% versus $12 \%$ ), although this difference was not statistically significant $(p=0.12)$.

Among HIV-infected women only, those with two or more HPV infections were more likely to have an associated Pap abnormality compared to those with zero or one HPV infection (33\% versus $10 \%, p=0.058)$. When eliminating those women without an HPV infection and comparing HIV-infected women with multiple infections to those with a single HPV infection, the difference is still substantial (33\% versus $20 \%)$ although not statistically significant $(p=$ 0.43 ). Thus, there may be an additive effect in which the combination of HIV infection and the presence of multiple concurrent HPV infections increases the association with cervical dysplasia greater than either risk factor alone.

Table 2 compares and summarizes the cross-sectional epidemiological differences in HPV and cervical dysplasia between HIV-infected and HIV-uninfected study participants. When focusing on HR-HPV infections, we found that, among HIV-infected women, there was a similar proportion of abnormal Pap tests results for those with one HR-HPV infection (38\%) and those with more than one $39 \%)(p$ $=0.94)$. Among HIV-uninfected women, the difference is greater (1 HR-HPV infection $=0 \%, 2$ or more HR-HPV infections $=50 \%)$ though not statistically significant due to the small size of this subset $(p=0.18)$.

Overall, our HIV-infected participants were not severely immune-compromised. Their average CD4 count was
$518 / \mathrm{mm}^{3}$ (IQR = 366-598; CD4 counts were not available for 10 participants). Forty-four percent were receiving antiretroviral (ARV) medications at the time of testing. The average CD4 count for those on ARV medications was 482 and for those not on ARV medications was $547(p=0.37)$. CD4 count was not found to be significantly associated with abnormal Pap test $(p=0.21)$ or total HPV infections $(p=0.07)$, although higher CD4 counts were associated with fewer HR-HPV infections $(r=-0.36, p=0.02)$. No significant relationship between ARV medications and the total number of HPV infections $(p=0.54)$ or the number of high-risk HPV infections ( $p=0.77$ ) was identified.

Our bivariate analyses identified positive associations between age and number of HPV infections (Pearson $r=$ $0.23, p=0.019$ ), number of HR-HPV infections (Pearson $r=$ $0.20, p=0.048$ ), and baseline Pap test abnormality (Spearman $r=0.20, p=0.050)$. There were no relationships observed between HPV infections or Pap abnormality and lifetime smoking, lifetime number of sexual partners, number of sexual partners in the last 6 months, and current use of condoms, birth control pills, or birth control injections (all $p$ values $>0.10$ )

\section{Discussion}

Our results demonstrate a large burden of HPV among our study population with a notably high prevalence of multiple concurrent infections. This is consistent with previous data from South Africa [16]. We confirmed previous work that identified a strong positive association between HIV and both $\mathrm{HPV}$ infection and HPV-related cervical dysplasia. Moreover, we found that the increased risks for cervical dysplasia conferred by HIV infection and multiple concurrent HPV infection may be additive. 
It is well established that HPV causes cervical cancer [17]. It is less clear, however, whether multiple concurrent HPV infections increase this risk. Some research has demonstrated an increased risk of carcinogenesis due to multiple concurrent infections compared to infection with a single HPV genotype $[10,18]$. One study identified a linear association between the number of HR-HPV infections and the severity of associated cervical dysplasia [9]. In a study of HIV-infected women, however, multiple concurrent infections were not found to have an increased association with cervical dysplasia [19]. We found a significantly positive association (twice the probability) between multiple concurrent infections and the risk of associated Pap smear abnormality.

Likewise, HIV infection is known to increase the risk of cervical dysplasia [2]. Data from the present study demonstrate HIV-infected participants to have a twofold greater proportion of Pap abnormalities compared to their HIVuninfected counterparts. Furthermore, when looking only at HIV-infected participants, a large but not statistically significant difference in association with Pap abnormality was found between those with a single HPV infection and those with multiple concurrent HPV infections, indicating a possible additive effect of HIV infections and infection with multiple concurrent HPVs in increasing the risk of cervical dysplasia.

The impact of multiple concurrent HPV infections on the duration of infection is uncertain. Some prior research has found no impact of multiple concurrent infections on HPV persistence $[20,21]$. In contrast, in a study of genotype specific duration of HPV infection, investigators found that coinfection with multiple HPV types increased the duration of infection for all HR-HPVs as a group as well as for HPV16 specifically [8]. The persistence of HPV infection is of critical importance as it has been demonstrated to increase the risk for incident precancerous lesions of the cervix [4, 22]. Due to sample size limitations, this study did not assess the impact of multiple concurrent HPV infections on HPV persistence.

This study has several limitations. The generalizability of the findings presented may be limited due to the specific geographical area from which the study cohort was drawn. Despite the numerous statistically significant findings, the relatively small sample size may have restricted the identification of important differences between groups and subgroups. Likewise, multivariate analyses examining differences between HIV-infected and HIV-uninfected participants while controlling for behavioral and demographic variables were not feasible due to the sample size. Lastly, because of the relative immune competency of our HIVinfected group and sample size limitations, study findings cannot be correlated with the extent of immunosuppression among HIV-infected participants.

\section{Conclusion}

In our cohort, Pap smear abnormalities were identified twice as frequently among HIV-infected women as among their $\mathrm{HIV}$-uninfected counterparts. In addition, among all women in the cohort, having multiple concurrent HPV infections was associated with twice the likelihood of an associated Pap smear abnormality compared to having a single HPV infection. These risk factors may be additive given our finding that HIV-infected women with multiple concurrent HPV infections were more likely to have associated Pap smear abnormalities than HIV-infected women with a single HPV infection.

\section{Conflict of Interests}

None of the authors have conflict of interests to disclose.

\section{Acknowledgment}

This work was supported by the National Institute of Allergy and Infectious Diseases at the National Institutes of Health (5 K23AI07759 to David Adler).

\section{References}

[1] G. M. Clifford, M. A. G. Gonçalves, and S. Franceschi, "Human papillomavirus types among women infected with HIV: a metaanalysis," AIDS, vol. 20, no. 18, pp. 2337-2344, 2006.

[2] L. S. Massad, L. Ahdieh, L. Benning et al., "Evolution of cervical abnormalities among women with HIV-1: evidence from surveillance cytology in the Women's Interagency HIV Study," Journal of Acquired Immune Deficiency Syndromes, vol. 27, no. 5, pp. 432-442, 2001.

[3] X.-W. Sun, T. V. Ellerbrock, O. Lungu, M. A. Chiasson, T. J. Bush, and T. C. Wright Jr., "Human papillomavirus infection in human immunodeficiency virus-seropositive women," Obstetrics and Gynecology, vol. 85, no. 5, part 1, pp. 680-686, 1995.

[4] N. F. Schlecht, S. Kulaga, J. Robitaille et al., "Persistent human papillomavirus infection as a predictor of cervical intraepithelial neoplasia," The Journal of the American Medical Association, vol. 286, no. 24, pp. 3106-3114, 2001.

[5] M. A. E. Nobbenhuis, J. M. M. Walboomers, T. J. M. Helmerhorst et al., "Relation of human papillomavirus status to cervical lesions and consequences for cervical-cancer screening: a prospective study," The Lancet, vol. 354, no. 9172, pp. 20-25, 1999.

[6] G. M. Clifford, S. Franceschi, O. Keiser et al., "Immunodeficiency and the risk of cervical intraepithelial neoplasia $2 / 3$ and cervical cancer: a nested case-control study in the Swiss HIV cohort study," International Journal of Cancer, vol. 138, no. 7, pp. 1732-1740, 2016.

[7] L. A. Denny, S. Franceschi, S. de Sanjosé, I. Heard, A. B. Moscicki, and J. Palefsky, "Human papillomavirus, human immunodeficiency virus and immunosuppression," Vaccine, vol. 30, supplement 5, pp. F168-F174, 2012.

[8] H. Trottier, S. Mahmud, J. C. M. Prado et al., "Type-specific duration of human papillomavirus infection: implications for human papillomavirus screening and vaccination," Journal of Infectious Diseases, vol. 197, no. 10, pp. 1436-1447, 2008.

[9] B. D. Bello, A. Spinillo, P. Alberizzi et al., "Cervical infections by multiple human papillomavirus (HPV) genotypes: prevalence and impact on the risk of precancerous epithelial lesions," Journal of Medical Virology, vol. 81, no. 4, pp. 703-712, 2009.

[10] H. Trottier, S. Mahmud, M. C. Costa et al., "Human papillomavirus infections with multiple types and risk of cervical 
neoplasia," Cancer Epidemiology Biomarkers and Prevention, vol. 15, no. 7, pp. 1274-1280, 2006.

[11] B. H. Yang, F. I. Bray, D. M. Parkin, J. W. Sellors, and Z.-F. Zhang, "Cervical cancer as a priority for prevention in different world regions: an evaluation using years of life lost," International Journal of Cancer, vol. 109, no. 3, pp. 418-424, 2004.

[12] F. X. Bosch, T. R. Broker, D. Forman et al., "Comprehensive control of human papillomavirus infections and related diseases," Vaccine, vol. 31, supplement 7, pp. H1-H31, 2013.

[13] K. S. Louie, S. De Sanjose, and P. Mayaud, "Epidemiology and prevention of human papillomavirus and cervical cancer in subSaharan Africa: a comprehensive review," Tropical Medicine \& International Health, vol. 14, no. 10, pp. 1287-1302, 2009.

[14] V. Bouvard, R. Baan, K. Straif et al., "A review of human carcinogens-part B: biological agents," The Lancet Oncology, vol. 10, no. 4, pp. 321-322, 2009.

[15] D. H. Adler, M. Wallace, T. Bennie et al., "Cervical dysplasia and high-risk human papillomavirus infections among HIVinfected and HIV-uninfected adolescent females in South Africa, Infectious Diseases in Obstetrics and Gynecology, vol. 2014, Article ID 498048, 6 pages, 2014.

[16] A. C. McDonald, A. I. Tergas, L. Kuhn, L. Denny, and T. C. Wright Jr., "Distribution of human papillomavirus genotypes among HIV-positive and HIV-negative women in Cape Town, South Africa," Frontiers in Oncology, vol. 4, article 48, 2014.

[17] F. X. Bosch, A. Lorincz, N. Muñoz, C. J. L. M. Meijer, and K. V. Shah, "The causal relation between human papillomavirus and cervical cancer," Journal of Clinical Pathology, vol. 55, no. 4, pp. 244-265, 2002.

[18] S. J. Patel, N. R. Mugo, C. R. Cohen et al., "Multiple human papillomavirus infections and HIV seropositivity as risk factors for abnormal cervical cytology among female sex workers in Nairobi," International Journal of STD and AIDS, vol. 24, no. 3, pp. 221-225, 2013.

[19] J. E. Levi, S. Fernandes, A. F. Tateno et al., "Presence of multiple human papillomavirus types in cervical samples from HIVinfected women," Gynecologic Oncology, vol. 92, no. 1, pp. 225231, 2004.

[20] N. G. Campos, A. C. Rodriguez, P. E. Castle et al., "Persistence of concurrent infections with multiple human papillomavirus types: a population-based Cohort Study," Journal of Infectious Diseases, vol. 203, no. 6, pp. 823-827, 2011.

[21] M.-C. Rousseau, J. S. Pereira, J. C. M. Prado, L. L. Villa, T. E. Rohan, and E. L. Franco, "Cervical coinfection with human papillomavirus (HPV) types as a predictor of acquisition and persistence of HPV infection," Journal of Infectious Diseases, vol. 184, no. 12, pp. 1508-1517, 2001.

[22] G. Y. F. Ho, R. D. Burk, S. Klein et al., "Persistent genital human papillomavirus infection as a risk factor for persistent cervical dysplasia," Journal of the National Cancer Institute, vol. 87, no. 18, pp. 1365-1371, 1995. 

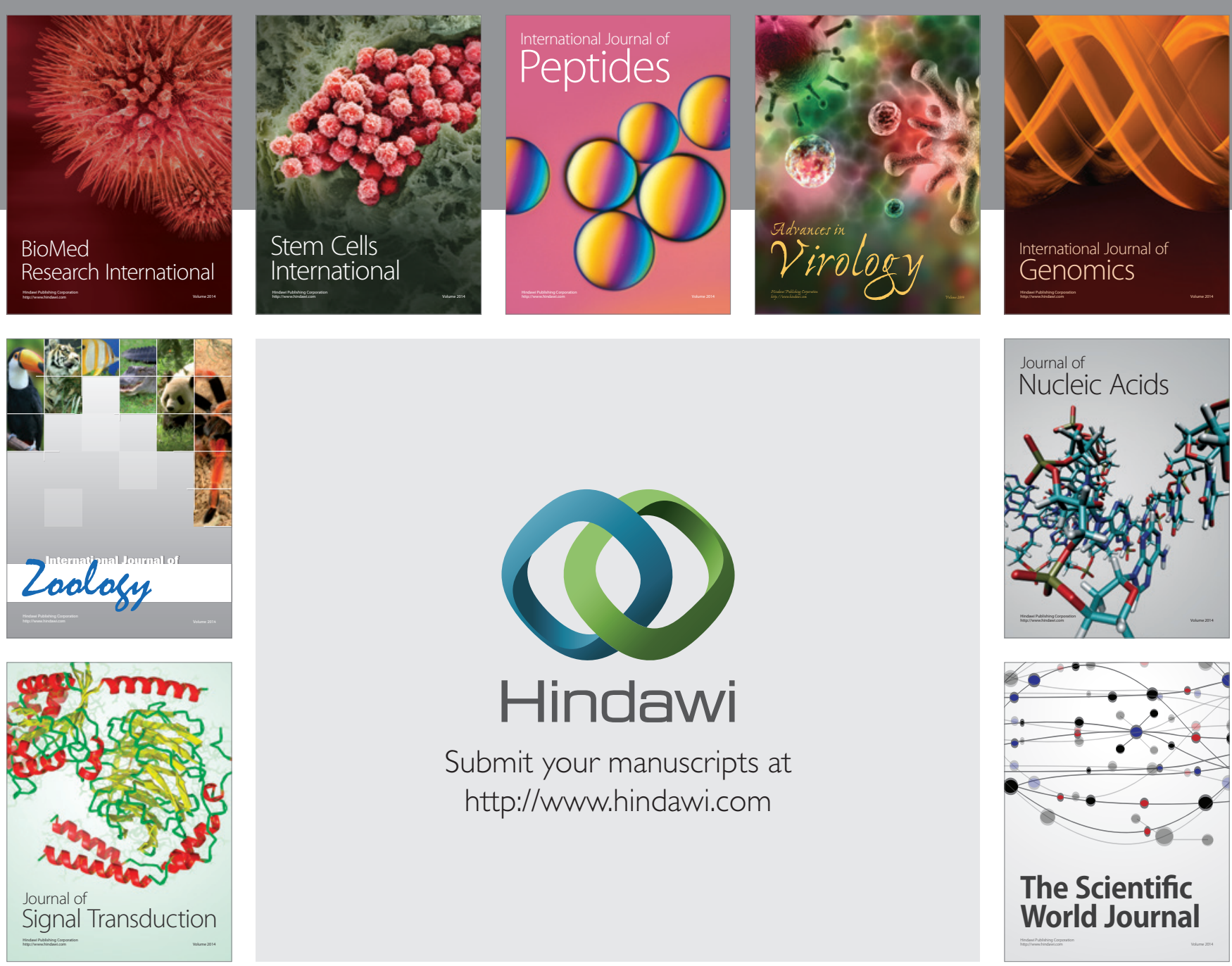

Submit your manuscripts at

http://www.hindawi.com
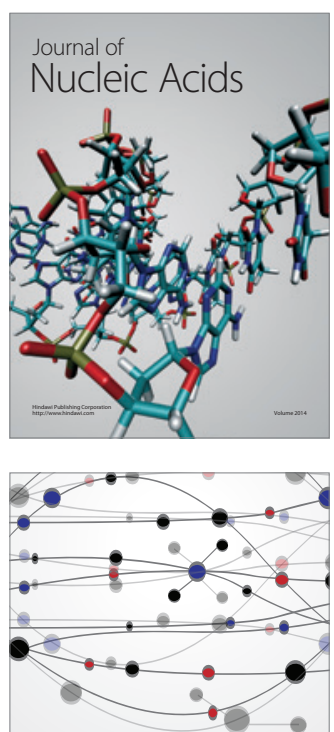

The Scientific World Journal
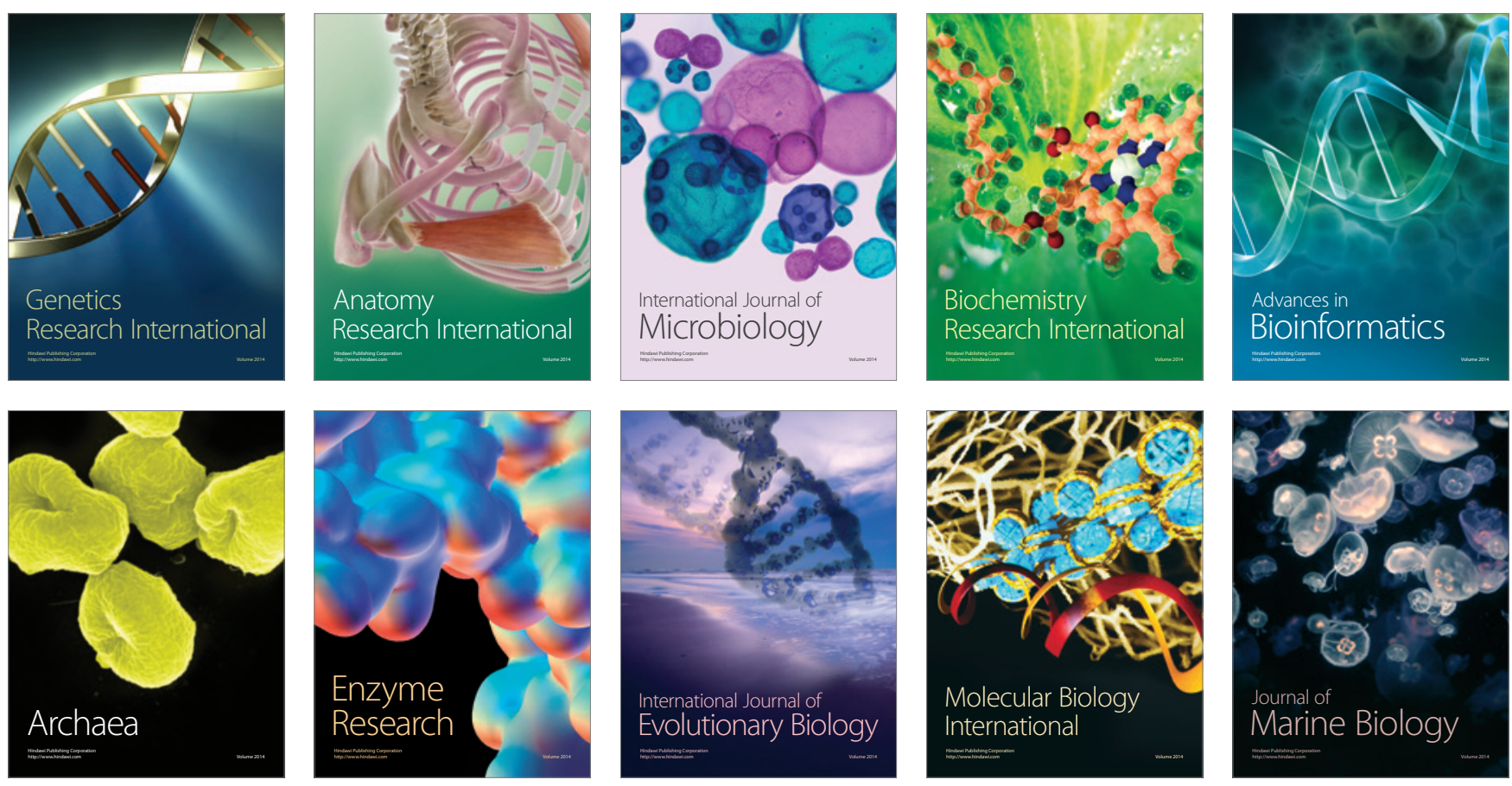